\title{
Aplicación de metodologías activas de aprendizaje en una nueva asignatura de Grado en Ingeniería Química
}

Beatriz Garcia-Fayos ${ }^{\mathrm{a}}$, Maria Sancho Fernandez ${ }^{\mathrm{b}}$ y Jose Miguel Arnal Arnalc

aUniversitat Politècnica de València. Departamento de Ingeniería Química y Nuclear. Camino de Vera s/n 46022 Valencia. E-mail: beagarfa@iqn.upv.es, bmsanchof@iqn.upv.es cjarnala@iqn.upv.es.

\begin{abstract}
Adaptation to the European Higher Education area has required the implementation of new teaching-learning strategies aimed to give more importance to the students and their involvement in their learning process. Two academic years ago, the implementation of the new plan's degree in Chemical Engineering presented us the need to design a new elective course in fourth year: Industrial Safety. The first year traditional methodology based on magistral lessons and problem resolution was followed with unsatisfactory results (academic and skills acquired by students). As a result, the second course was raised a new strategy based on active methodologies to improve the subject, increase student motivation and involve them in a more active learning. Contents were selected and structured, some of them linked with a previous core subject, activities have been designed to encourage student participation in class, based on observation and analysis of real situations, as well as law application. This paper shows the satisfactory results of the evaluation of the new methodology by the students, regarding contents, activities and learning degree of certain skills acquired as a result of the course. The main conclusions will be taken as reference in order to continue Safety learning using active methodologies.
\end{abstract}

Keywords: Safety, Chemical engineering education, active learning

\begin{abstract}
Resumen
La adaptación al Espacio Europeo de Educación Superior ha requerido la implementación de estrategias de enseñanza y aprendizaje que den más protagonismo al alumno y a su implicación. Hace dos cursos académicos, como consecuencia del nuevo plan de Grado en Ingeniería Química, nos vimos en la necesidad de diseñar una nueva asignatura de cuarto curso:
\end{abstract}


Aplicación de metodologías activas de aprendizaje en una nueva asignatura de Grado en Ingeniería Química

Seguridad Industrial. El primer curso académico se siguió la metodología tradicional con clases magistrales y resolución de problemas con resultados poco satisfactorios tanto en nivel de los resultados, como habilidades adquiridas. El segundo segundo curso académico, se planteó una nueva estrategia basada en la introducción de metodologías activas para mejorar la asignatura. Se seleccionaron y estructuraron los contenidos, vinculándolos con una asignatura troncal previa, se diseñaron actividades para aumentar la participación en las clases, basándose en el análisis y observación de situaciones reales, así como en uso de legislación vigente. Este trabajo muestra los resultados satisfactorios del cambio de metodología obtenidos de la valoración de contenidos, actividades y metodología implementada y el grado de aprendizaje adquirido como consecuencia de la asignatura realizado por los alumnos. Las principales conclusiones serán tomadas como punto de partida para continuar con la enseñanza de Seguridad.

\section{Palabras clave:Seguridad, Educación en Ingeniería Química, Aprendizaje activo.}

\section{Introducción}

En el campo de la enseñanza en Ingeniería Química, y en concreto en Seguridad, existe un acuerdo común en afirmar que la enseñanza en esta disciplina debe ser obligatoria en todos los cursos de Ingeniería Química (Pitt, 2012; Shallcross, 2013), como una herramienta para prevenir accidentes, y en respuesta al papel relevante que el Ingeniero Químico posee a la hora de prevenir y reducir el riesgo asociado a los accidentes que pueden ocurrir en una planta o actividad industrial (Darbra, 2012).

La enseñanza-aprendizaje en Seguridad debe ser considerada no como un complemento de la enseñanza de procesos industriales sino como una parte integral del curriculum de la ingeniería (Hendershot, 2007).

La introducción de esta disciplina en el curriculum del Ingeniero Químico ha sido objeto de reflexión y discusión durante años (Harvey, 1984; Kletz, 1988; Fleischman, 1988) sobre su entidad para introducirla como asignatura o como una materia adicional en diferentes asignaturas (Lane, 1989), no existiendo acuerdo todavía (Shallcross, 2014). En los últimos años, a raíz del reconocimiento como requisito de esta disciplina por organismos tales como "Institution of Chemical Engineers" o el "International Engineering Alliance" o de 
acreditación como "Accreditation Board for Engineering and Technology", ABET (Shallcross, 2014), se ha ido extendiendo su inclusión como asignatura propia en el curriculum del Ingeniero Químico, puesto que mejora la coherencia y coordinación de los contenidos, además de ser impartida de forma sistemática y por profesorado con el suficiente interés, conocimiento y experiencia (Perrin, 2008).

Respecto a la metodología de impartición, un sistema tradicional de enseñanza en el que se incluyan instrucciones, contexto histórico y una explicación de las interacciones físicasquímicas y la protección frente a estas, proporciona un enfoque limitado con objeciones de tipo pedagógico (ya que no hay preguntas, solo respuestas prefabricadas que no permiten el pensamiento creativo sobre seguridad), y de tipo técnico (ya que no se realiza una identificación de alternativas, comparación, selección y evaluación de éstas) (Ferjencik, 2007; Darbra, 2012).

Si se busca formar futuros profesionales capaces de identificar, seleccionar, evaluar y comparar las distintas alternativas para resolver problemas de seguridad durante su vida laboral de forma independiente y óptima. Para ello se precisa una enseñanza-aprendizaje basada en el razonamiento (Ferjencik, 2007) y en un papel activo de los alumnos más cercano al rol profesional que desarrollaran, y los profesores debemos proporcionar las herramientas que permitan que esto suceda.

La actual situación de la enseñanza universitaria adaptada a Bolonia y al Espacio Europeo de Educación Superior proporciona el marco adecuado para que la enseñanza-aprendizaje de la Seguridad pueda desarrollarse de forma útil para los futuros profesionales, ya que potencia el protagonismo del alumno y su implicación en dicho proceso.

Una de las estrategias que se pueden implementar es el uso de metodologías activas, entendidas como aquellos métodos, técnicas y estrategias que utiliza el docente para convertir el proceso de enseñanza en actividades que fomenten la participación activa del estudiante y lleven al aprendizaje (Labrador, 2008). Existen algunas experiencias documentadas sobre el uso de estas metodologías utilizadas en la enseñanza-aprendizaje de Seguridad Industrial para Ingenieros Químicos con buenos resultados.

Las metodologías citadas son el estudio de caso basándose en análisis de accidentes reales en una asignatura de "Introducción a la seguridad" de Grado (Ferjencik, 2007), o como seminarios en asignaturas de "Análisis de procesos Químicos 1 y 2 ” (Shallcross, 2013). Esta metodología permite trabajar en mayor profundidad los contenidos, así como trabajar otras competencias tales como la presentación oral y habilidades de comunicación a la hora de presentar y defender los casos estudiados.

Hay experiencias que han propuesto trabajar esta disciplina a través de "role-playing", utilizando casos reales en los que los alumnos actúan como "expertos" o asesores de 
Aplicación de metodologías activas de aprendizaje en una nueva asignatura de Grado en Ingeniería Química

seguridad que investigan un accidente, explicando el caso, las causas y su solución (Darbra, 2012). Esta metodología aumenta el interés y la implicación del alumnado, ya que sienten que están trabajando en un caso real. Además, permite desarrollar la capacidad de trabajo en equipo y la creatividad.

Otros autores proponen los "safety shares", entendidos como discusión de 2-4 minutos sobre algún aspecto de seguridad, tal y como se hace en empresas reales (Shallcross, 2014) en las que se tiene implantada una verdadera cultura de seguridad y prevención de riesgos laborales.

Finalmente, se tiene experiencias sobre aprendizaje de seguridad en asignaturas de experimentación que se imparten en laboratorios (Peñas 2006; Arnal, 2009), dado que proporcionan un entorno real donde se pueden poner en práctica muchos de los contenidos relacionados con la seguridad y la concienciación sobre su importancia a través de la práctica, autoaprendizaje y entrenamiento (Abu-Khalaf, 2001). Están documentadas metodologías de “role-playing” o simulación (Graells, 2007),(Garcia-Fayos, 2012) y de aprendizaje basado en proyectos (Sancho, 2007) utilizando el laboratorio como escenario real de aprendizaje.

El presente trabajo describe la experiencia llevada a cabo por un grupo de profesores que imparten la asignatura de Seguridad Industrial en el Grado de Ingeniería Química, que previamente habían iniciado la aplicación de metodologías activas para impartir esta disciplina de forma transversal en asignaturas experimentales de Ingeniería Química del plan de 1997 (Sancho, 2007; Arnal, 2009; Garcia-Fayos, 2012) y de Grado en Ingeniería Química (Sancho, 2015).

\section{Objetivo}

El objetivo es exponer los resultados del cambio de metodología de trabajo en la asignatura optativa “Seguridad Industrial” del Grado en Ingeniería Química, y valorar si la implantación de una metodología activa en el aula consigue aumentar la motivación y la implicación de los alumnos en esta materia, así como el grado de aprendizaje de determinadas competencias que permitan una mejor preparación en este ámbito de los futuros profesionales.

Los indicadores objetivamente verificables que permitirán valorar si el cambio realizado ha logrado este propósito, serán la valoración de la asignatura a través de encuesta anónima a los alumnos, y la valoración cualitativa del profesorado tras la implantación del cambio de metodología. 


\section{Contexto, motivación y desarrollo de la innovación educativa}

La innovación educativa se ha llevado a cabo en la asignatura Seguridad Industrial, una nueva asignatura optativa de la Mención "Diseño y Seguridad Industrial" perteneciente al cuarto curso, cuatrimestre B, en el Grado de Ingeniería Química de la Universitat Politècnica de València.

El curso académico de su creación (2013-2014) supuso un reto para los profesores, puesto que esta disciplina no había sido incluida en los planes de estudio previos de Ingeniería Química en esta Universidad. Únicamente se había impartido de forma transversal en asignaturas experimentales troncales de esta titulación, aprovechando el escenario real que presentaba la manipulación de sustancias químicas y equipos en el laboratorio (Sancho, 2015).

El primer curso académico, se optó por una metodología tradicional, basada en lecciones magistrales y resolución de problemas, complementadas con una visita de campo a empresa. La principal dificultad fue la selección de contenidos y la creación de los materiales para su impartición, dado que los conocimientos previos de los alumnos en esta materia eran básicos.

Los resultados de este primer curso fueron poco satisfactorios, al no alcanzar las expectativas de la asignatura ni por parte de los profesores ni de los alumnos, tanto a nivel de los resultados académicos como de las habilidades adquiridas. En conversaciones informales, los alumnos manifestaron la necesidad de adquirir conocimientos, pero también de aplicarlos en situaciones y procesos concretos como un acercamiento al desarrollo de su actividad profesional futura.

Como consecuencia, en el siguiente curso académico decidimos replantear la estrategia docente en la asignatura, abordando los aspectos que se desarrollan a continuación.

\subsection{Selección y reestructuración de los contenidos}

En lugar de impartir los contenidos de forma monográfica e indepediente, tal y como se hizo en el curso 2013-2014, se seleccionaron y reestructuraron los contenidos vinculándolos a un proceso industrial previamente descrito en la asignatura troncal de cuarto curso "Procesos Industriales en Ingeniería Química" impartida en el cuatrimestre A. De esta forma, se pretendía que los alumnos aplicaran los contenidos de la asignatura de Seguridad Industrial a procesos previamente descritos y conocidos, de forma que además pudieran relacionar los conceptos de ambas asignaturas.

La tabla 1 muestra los 12 temas impartidos en el curso 2013-2014 y los 10 temas del curso 2014-2015. El número de temas se vio reducido con el fin de trabajar con mayor profundidad los contenidos más significativos, ampliando incluso sesiones en el caso de la

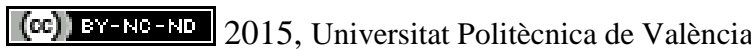


Aplicación de metodologías activas de aprendizaje en una nueva asignatura de Grado en Ingeniería Química

"Identificación y evaluación de riesgos" y en el caso de los "Equipos de protección individual (EPI’s)" y "Primeros Auxilios".

Tabla 1. Selección de temas en la asignatura de Seguridad Industrial

\begin{tabular}{cccc}
\hline Tema & Curso 2013-2014 & Tema & Curso 2014-2015 \\
\hline 1 & Introducción a la Seguridad & 1 & Introducción a la seguridad \\
2 & Agentes físicos, químicos y biológicos & 2 & Ficha de Seguridad \\
3 & Enfermedades profesionales & 3 & Agentes físicos, químicos y \\
biológicos
\end{tabular}

\subsection{Diseño de las actividades}

A diferencia del curso anterior, en este curso se ha aumentado el número de actividades y se han diseñado algunas nuevas con el fin de fomentar la participación de los estudiantes en las clases. Así, se han incluído actividades como:

- observación y análisis de situaciones reales (visitas a empresa y casos reales basados en accidentes recientes o en noticias)

- $\quad$ actividades de "role-playing" (ejerciendo como inspectores de seguridad o como trabajadores en un puesto de trabajo concreto), con el fin de que los alumnos 
evaluaran la situación y tomaran decisiones para identificar problemas y aportar soluciones

- actividades de aplicación de legislación en materia de Seguridad

- visitas de campo, en las que los estudiantes pudieron observar situaciones reales y han tenido que aplicar los conocimientos adquiridos para realizar propuestas de mejora.

La tabla 2 muestra las actividades realizadas en ambos cursos académicos.

Tabla 2. Actividades en la asignatura de Seguridad Industrial

\begin{tabular}{|c|c|}
\hline Curso 2013-2014 & Curso 2014-2015 \\
\hline Resolución de problemas & A Resolución encuesta de Seguridad inicial \\
\hline \multirow[t]{6}{*}{ Visita a empresa } & B Visita como inspectores de seguridad a un laboratorio \\
\hline & C Resolución, puesta en común y corrección de la inspección \\
\hline & D Visitas de campo \\
\hline & $\begin{array}{l}\text { E Resolución, puesta en común y corrección del informe de la } \\
\text { visita }\end{array}$ \\
\hline & $\begin{array}{c}\text { F “Role-playing” para la identificación de riesgos en puestos } \\
\text { trabajo de un proceso }\end{array}$ \\
\hline & $\begin{array}{c}\text { G "Role-playing” para la propuesta de medidas preventivas en } \\
\text { puestos trabajo de un proceso }\end{array}$ \\
\hline
\end{tabular}

La participación en las dinámicas propuestas se ha realizado de forma grupal, con 4 grupos de 4-5 alumnos, así como de forma individual (en el caso de Resolución de la encuesta y las visitas a empresa).

\subsection{Metodología desarrollada}

La metodología tradicional basada en lección magistral para el desarrollo de los contenidos teóricos se ha cambiado por una basada en la aplicación de metodologías activas, que fomentan la implicación y participación del alumnado.

La tabla 3 muestra las metodologías implementadas y su relación con los contenidos y con las actividades desarrolladas en el curso académico 2014-2015. 
Aplicación de metodologías activas de aprendizaje en una nueva asignatura de Grado en Ingeniería Química

En la tabla se observa que las principales metodologías utilizadas han sido el método del caso, el aprendizaje cooperativo, la lección magistral participativa y el "role-playing” o simulación.

Se observa también que en la impartición de algunos de los contenidos, como la introducción a la seguridad o la identificación y evaluación de riesgos, se han utilizado distintas metodologías.

Tabla 3. Relación metodología, contenidos y actividades en la asignatura de Seguridad industrial en el curso 2014-2015

\begin{tabular}{|c|c|c|}
\hline Metodología & Contenidos & Actividades \\
\hline $\begin{array}{c}\text { Método del caso } \\
\text { Aprendizaje cooperativo }\end{array}$ & 1. Introducción a la seguridad & $\begin{array}{l}\text { Resolución encuesta de } \\
\text { Seguridad inicial }\end{array}$ \\
\hline Lección magistral participativa & 2. Ficha de Seguridad & --- \\
\hline Aprendizaje cooperativo & $\begin{array}{l}\text { 3. Agentes físicos, químicos y } \\
\text { biológicos }\end{array}$ & --- \\
\hline Lección magistral participativa & $\begin{array}{l}\text { 4. Técnicas de ventilación y } \\
\text { extracción localizada }\end{array}$ & --- \\
\hline Lección magistral participativa & 7. Equipos a presión & $\begin{array}{c}\text { Clasificación de los equipos } \\
\text { de acuerdo a legislación }\end{array}$ \\
\hline Role-playing o simulación & 5. Identificación de riesgos & $\begin{array}{l}\text { Visita como inspectores de } \\
\text { seguridad a un laboratorio }\end{array}$ \\
\hline \multirow[t]{4}{*}{ Aprendizaje cooperativo } & $\begin{array}{c}\text { 6. Evaluación de riesgos y } \\
\text { propuesta de medidas } \\
\text { preventivas }\end{array}$ & $\begin{array}{l}\text { Resolución, puesta en común } \\
\text { y corrección de la inspección }\end{array}$ \\
\hline & & Visita a empresa \\
\hline & & $\begin{array}{l}\text { Resolución, puesta en común } \\
\text { y corrección informe visita }\end{array}$ \\
\hline & & $\begin{array}{c}\text { “Role-playing” para } \\
\text { identificación riesgos y } \\
\text { medidas preventivas }\end{array}$ \\
\hline Lección magistral participativa & 8 Amianto & --- \\
\hline \multirow[t]{2}{*}{ Role-playing o simulación } & 9 EPI’s & --- \\
\hline & 10 Primeros auxilios & --- \\
\hline
\end{tabular}




\subsection{Competencias}

Las metodologías aplicadas están orientadas a trabajar una serie de competencias (tanto transversales como específicas) que se desea que adquieran los alumnos al finalizar la asignatura.

La tabla 4 muestra la relación entre competencias y los contenidos-actividades realizadas. Las competencias se han definido de acuerdo con el proyecto institucional de la UPV de competencias transversales (UPV, 2015) que se encuadran en el Plan estratégico UPV 2015-2020.

Tabla 4. Relación de competencias con los contenidos y actividades trabajadas en la asignatura de Seguridad industrial en el curso 2014-2015

\begin{tabular}{|c|c|c|}
\hline Contenidos & Actividades & Competencia \\
\hline \multirow[t]{3}{*}{ Introducción a la seguridad } & $\begin{array}{l}\text { Resolución encuesta } \\
\text { de Seguridad inicial }\end{array}$ & $\begin{array}{c}\text { A Pensamiento crítico (Por ejemplo: } \\
\text { identificar riesgos de un puesto de } \\
\text { trabajo y priorizar medidas } \\
\text { preventivas) }\end{array}$ \\
\hline & & $\begin{array}{c}\text { B Comprensión e integración (de } \\
\text { situaciones actuales relacionadas con la } \\
\text { Seguridad Industrial) }\end{array}$ \\
\hline & & $\begin{array}{c}\text { C Conocimiento de problemas } \\
\text { contemporáneos (por ejemplo: } \\
\text { interpretación de noticias, casos reales, } \\
\text { etc.) }\end{array}$ \\
\hline Ficha de Seguridad & --- & D Manejo de legislación (específica) \\
\hline $\begin{array}{l}\text { Agentes físicos, químicos y } \\
\text { biológicos }\end{array}$ & --- & $\begin{array}{c}\text { B Comprensión e integración (de } \\
\text { situaciones actuales relacionadas con la } \\
\text { Seguridad Industrial) } \\
\text { C Conocimiento de problemas } \\
\text { contemporáneos (por ejemplo: } \\
\text { interpretación de noticias, casos reales, } \\
\text { etc.) }\end{array}$ \\
\hline $\begin{array}{l}\text { Técnicas de ventilación y } \\
\text { extracción localizada }\end{array}$ & --- & $\begin{array}{c}\text { A Pensamiento crítico (Por ejemplo: } \\
\text { identificar riesgos de un puesto de } \\
\text { trabajo y priorizar medidas } \\
\text { preventivas) }\end{array}$ \\
\hline Equipos a presión & --- & D Manejo de legislación (específica) \\
\hline
\end{tabular}

(c) EY-NC-ND 2015, Universitat Politècnica de València

Congreso In-Red (2015) 
Aplicación de metodologías activas de aprendizaje en una nueva asignatura de Grado en Ingeniería Química

\begin{tabular}{|c|c|c|}
\hline $\begin{array}{l}\text { Identificación de riesgos } \\
\text { Evaluación de riesgos y } \\
\text { propuesta de medidas } \\
\text { preventivas }\end{array}$ & $\begin{array}{c}\text { Visita inspectores } \\
\text { seguridad } \\
\text { Resolución, puesta en } \\
\text { común y corrección } \\
\text { inspección } \\
\text { Visita a empresa } \\
\text { Resolución, común y } \\
\text { corrección visita } \\
\text { "Role-playing” } \\
\text { pidentificación } \\
\text { Riesgos y medidas } \\
\text { preventivas }\end{array}$ & $\begin{array}{l}\text { A Pensamiento crítico (Por ejemplo: } \\
\text { identificar riesgos de un puesto de } \\
\text { trabajo y priorizar medidas preventivas) } \\
\text { E Aplicación del pensamiento práctico } \\
\text { (por ejemplo: identificar el montaje y los } \\
\text { riesgos de los productos químicos, } \\
\text { procesar información de la FDS y lo } \\
\text { observado y elaborar la lista de medidas } \\
\text { preventivas para minimizar los riesgos) }\end{array}$ \\
\hline Amianto & --- & D Manejo de legislación (específica) \\
\hline $\begin{array}{c}\text { EPI's } \\
\text { Primeros auxilios }\end{array}$ & $\begin{array}{l}--- \\
---\end{array}$ & $\begin{array}{c}\text { A Pensamiento crítico (Por ejemplo: } \\
\text { identificar riesgos de un puesto de } \\
\text { trabajo y priorizar medidas } \\
\text { preventivas) }\end{array}$ \\
\hline
\end{tabular}

\section{Resultados}

La valoración de la innovación educativa realizada se ha llevado a cabo de dos formas:

A) A través de encuesta anónima, los alumnos valoraron los contenidos de la asignatura (en cada uno de los 10 temas indicados en la Tabla 1), las actividades realizadas (señaladas en la Tabla 2), y el grado de aprendizaje de determinados items, de acuerdo con la escala y las cuestiones que se indican en la tabla 5.

Tabla 5. Modelo de encuesta de valoración de la asignatura realizada por los alumnos

Valora los temas impartidos y las actividades realizadas, teniendo en cuenta la siguiente escala:

Muy Bien (1); Bien (2); Regular (3); Mejorable (4)

Indica el grado de aprendizaje como consecuencia en esta asignatura en determinados items:

Nada (1); Poco (2); Bastante (3); Mucho (4)

La tabla 6 muestra los ítems preguntados a los alumnos en la última parte de la encuesta, y su relación con las competencias del Plan UPV. 
B. Garcia-Fayos, M. Sancho Fernandez, J.M. Arnal Arnal

Tabla 6. Relación ítem-competencia UPV

\begin{tabular}{cc}
\hline Item preguntado & Competencia UPV \\
\hline $\begin{array}{c}\text { Ante una situación real proponer soluciones } \\
\text { técnicas con juicio crítico }\end{array}$ & Pensamiento crítico \\
$\begin{array}{c}\text { Identificación y comprensión de situaciones } \\
\text { actuales relacionadas con la Seguridad } \\
\text { Industrial }\end{array}$ & Comprensión e integración \\
Toma de decisiones & Conocimiento de problemas contemporáneos \\
Manejo de legislación & Aplicación y pensamiento práctico \\
\end{tabular}

B) A partir de la valoración cualitativa de los profesores en las reuniones de coordinación de la asignatura.

\subsection{Resultados de valoración por parte de los alumnos}

Los resultados que se presentan a continuación se corresponden con la valoración de los contenidos de la asignatura.

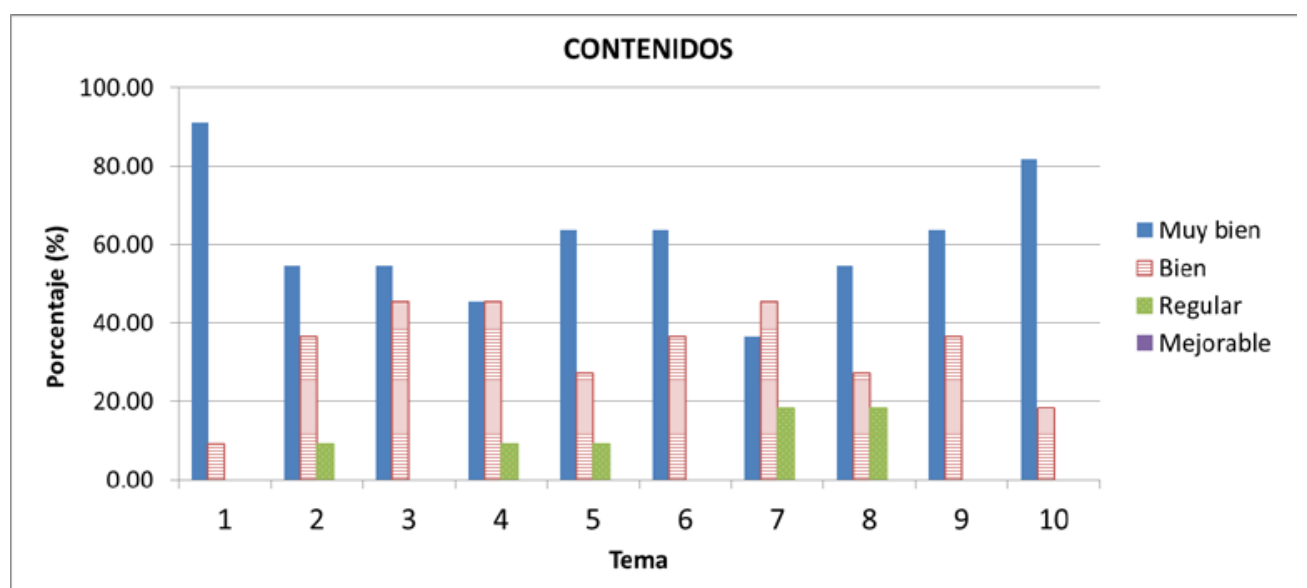

Fig. 1 Evaluación de los contenidos de la asignatura Seguridad Industrial

Como se observa en la Fig. 1, cinco de los diez temas (Temas 1, 3, 6 , 9 y 10) han sido valorados como "Muy bien" o "Bien" por el $100 \%$ de los alumnos; tres de los diez temas han sido valorados como "Muy bien" o "Bien" por el $90.9 \%$ de los alumnos (Temas 2, 4 y 5); y dos temas como "Muy bien" o "Bien" por el $80.8 \%$ de los alumnos (Temas 7 y 8).

(c)) EY-NC-ND 2015, Universitat Politècnica de València

Congreso In-Red (2015) 
Aplicación de metodologías activas de aprendizaje en una nueva asignatura de Grado en Ingeniería Química

Cabe destacar que que los temas mejor valorados por los alumnos han sido aquéllos en los que se han aplicado metodologías activas de aprendizaje cooperativo y simulación o "roleplaying”.

La Fig. 2 muestra los resultados de valoración de las actividades realizadas por los alumnos.

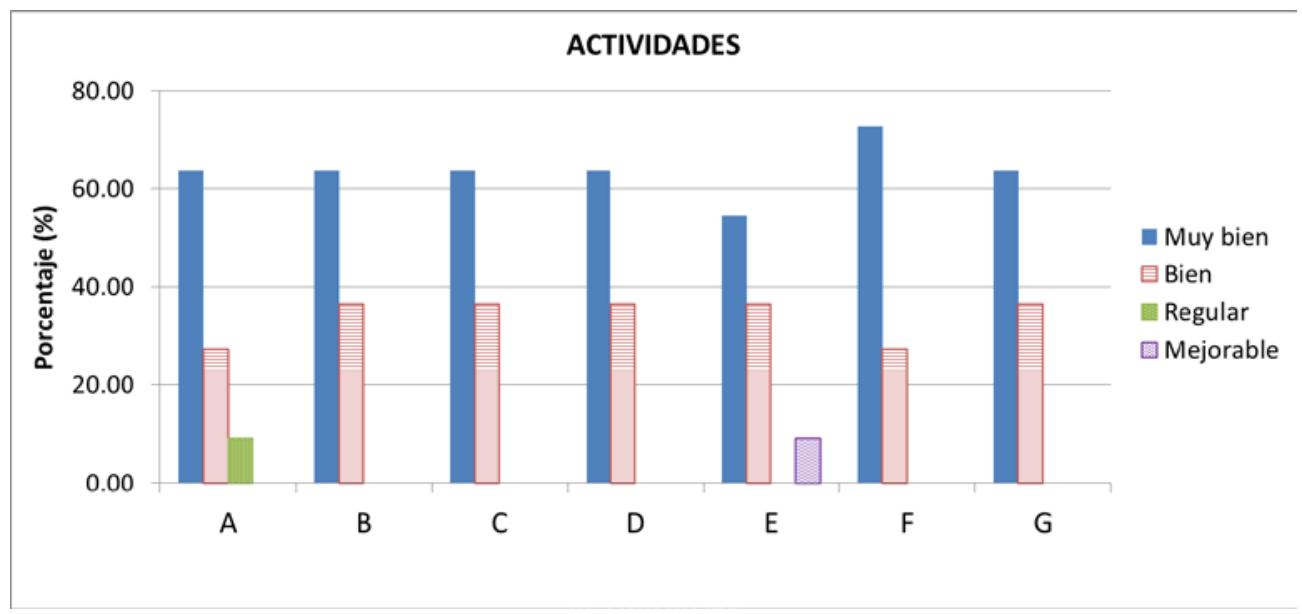

Fig. 2 Evaluación de las actividades de la asignatura Seguridad Industrial

Como se observa, 5 de las 7 actividades han sido valoradas como "Muy bien" o "Bien" por el 100 \% de los alumnos, especialmente las de "Role-playing" (Actividades F y G). Las 2 restantes (Resolución encuesta-actividad A y Resolución y puesta en común informe visitaactividad E) como "Muy bien" o "Bien" por el 90.9 \% de los alumnos, coincidentes ambas con actividades de resolución y puesta en común de actividades planteadas. A pesar de estar estas dos ultimas peor valoradas por los alumnos, están relacionadas con los temas 1 y 6 que son lo que mejores resultados obtuvieron al ser valorados sus contenidos. Por tanto, parece que a la hora de realizar la valoración, influye más el tipo de actividad en sí que el contenido que se esté tratando.

La Fig. 3 muestra los resultados de la valoración realizada por los alumnos del grado de aprendizaje en determinadas competencias. 


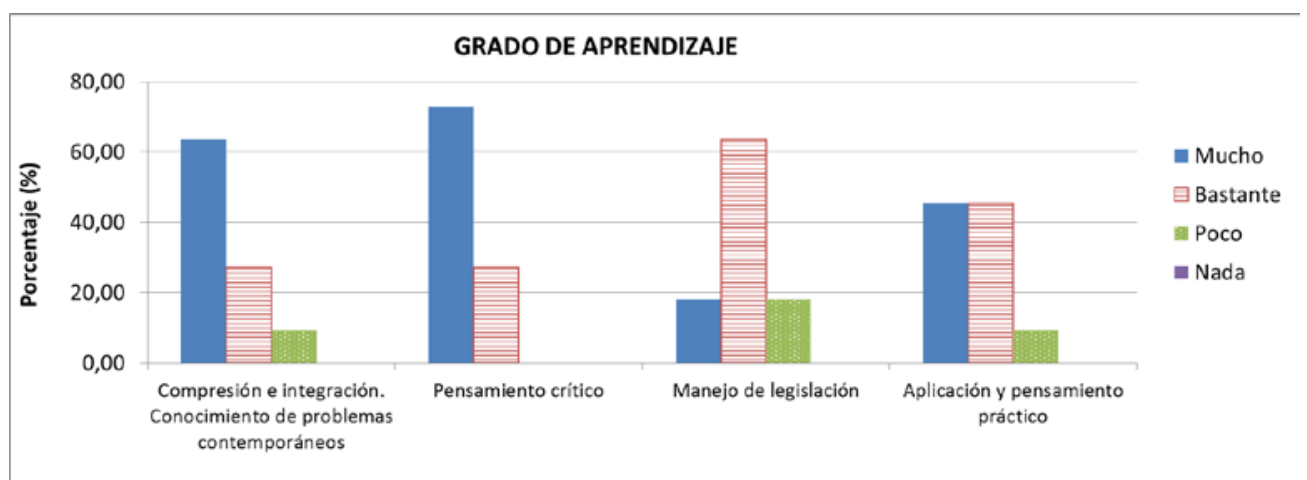

Fig. 3 Evaluación del grado de aprendizaje de las competencias de la asignatura Seguridad Industrial

Tal y como se observa en la Figura 3, los alumnos consideran que la asignatura les ha ayudado mucho a mejorar el "pensamiento crítico", bastante o mucho para el $100 \%$ de los alumnos. Por otra parte, en los ítems correspondientes a las competencias: "comprensión e integración”, "conocimiento de problemas contemporáneos” y "aplicación y pensamiento práctico", el $90.91 \%$ de los alumnos estiman que su grado de aprendizaje ha mejorado mucho o bastante. Finalmente, el "Manejo de legislación" es la competencia en la que menor porcentaje de alumnos perciben que han mejorado.

\subsection{Valoración cualitativa del profesorado}

La valoración del profesorado del cambio de metodología en las distintas reuniones de coordinación mantenidas durante el cuatrimestre del curso 2014-2015 ha sido muy satisfactoria. Se percibía un aumento de la motivación del alumnado, interesándose, mientras se impartía la asignatura, por noticias recientes que sucedían en relación a accidentes o fallos de seguridad basados en experiencias propias o del entorno cercano. También se ha percibido una mayor participación del alumnado en clase. Dado que era un grupo de 18 alumnos, se conseguía que todos los alumnos participaran en las discusiones y aportaran ideas en todas las sesiones. Aunque el planteamiento de cambiar la asignatura ha supuesto mucho trabajo y una gran dedicación, los profesores estamos satisfechos con los resultados obtenidos y la respuesta de los alumnos en la asignatura, por lo que se ha acordado repetir la misma estrategia para el curso siguiente.

\section{Conclusiones}

Las principales conclusiones de este trabajo son las siguientes :

-Para lograr un aprendizaje efectivo y la adquisición de competencias que el alumno vaya a aplicar en su vida profesional real es necesaria la motivación e implicación del alumnado.

-El uso de metodologías activas como el "role-playing", o el aprendizaje cooperativo son herramientas útiles para lograr dicho objetivo.

\section{(c) EY-NC-ND 2015, Universitat Politècnica de València}


Aplicación de metodologías activas de aprendizaje en una nueva asignatura de Grado en Ingeniería Química

-La selección adecuada de contenidos técnicos y el diseño de actividades con enfoque práctico y aplicado, así como la colaboración y participación del alumnado, son las claves fundamentales para alcanzar el éxito del proyecto.

-Los alumnos valoran de forma muy positiva tanto los contenidos como las actividades realizadas, y opinan que como consecuencia de la asignatura han logrado una mejora notable en las competencias de pensamiento crítico, comprensión e integración, conocimiento de problemas contemporáneos y aplicación y pensamiento práctico.

\section{Referencias}

ARNAL, J.M.; SANCHO, M.; GARCÍA-FAYOS B. (2009). "The formation in security and health of the second cycle students of the technical degrees”. Gomez-Chova L., Martí-Belenguer D., CandelTorres, I.(ed.) . En International Technology, Education and Development Conference-INTED 2009 (Marzo. 2009). Valencia: IATED. 2855-2860.

DARBRA, R.M.; CASAL, J.; PASTOR, E., VILCHEZ J.A.; ARNALDOS, J.; PLANAS E. (2012). "Risk analysis active learning through the investigation of real cases" en Process Safety and Environmental Protection, 90, 430.

FERJENCIK, M. (2007). “Best starting point to comprehensive process Safety education” en Process Safety Progress, 26,3,195.

FLEISCHMAN, M. (1988). "Rationale for incorporating health and safety into the curriculum” en Chemical Engineering Education, Winter, 30.

GARCÍA-FAYOS, B.; SANCHO, M.; ARNAL, J.M. (2012). “Análisis de la implantación de la metodología de "Role-playing” en una asignatura experimental de Ingeniería Química ”. Instituto de Ciencias de la Educación ICE-UPV. En: Jornada de Innovación Docente ICE-UPV 2012 (Julio. 2012). Valencia: Editorial UPV. 111-115.

GRAELlS, M.; PÉREZ-MOYA, M. (2007) “Projecte PEEEQ: Planificació estratégica de les asignatures d'Experimentació en Enginyeria Química de l'Escola Universitària d'Enginyeria Tècnica Industrial de Barcelona”. Universitat Politécnica de Catalunya, 2007.

HARVEY, B.H. (1984). “Third Report of the Advisory Committee on Major Hazards”. H.M. Stationery Office, London, UK.

HENDERSHOT, D.C. y SMADES W.(2007). "Safety culture begins in the classroom” en Process Safety Progress, 26, 2, 83.

KLETZ, T.A. (1988). "Should undergraduates be instructed in loss prevention?" en Plant and Operation Progress, 7, 2, 95.

LABRADOR PIQUER, M.J. y ANDREU ANDRES, M.A. (2008). Metodologías activas. Grupo de innovación en metodologías activas (GIMA). Valencia: Editorial Universitat Politècnica de valència

LANE, A.M. (1989) "Incorporating health, safety, environmental and ethical issues into the curriculum en Chemical Engineering Education, Spring, 70. 
PEÑAS F.J, BARONA A., ELIAS A., OLAZAR M. (2007). "Implementation of industrial health and safety in chemical engineering teaching laboratories“ en Journal of Chemical Health \& Safety, Marc/April, 19.

PERRIN, L., LAURENT A. (2008). "Current Situation and future implementation of safety curricula for chemical engineering education in France” en Education for Chemical Engineers, 3, e84.

PITT, M.J. (2012). “Teaching safety in Chemical Engineering. What, How and Who?” en Chemical Engineering and Technology, 35, 8, 1341.

SANCHO, M.; ARNAL, J.M.; SANTAFÉ, A.; CUARTAS, B.; MARTÍNEZ, F.; GARCÍA-FAYOS, B. (2007) "A new methodology in an experimental subject of chemical engineering”. Gomez-Chova L., Martí-Belenguer D., Candel-Torres, I.(ed.) . En International Technology, Education and Development Conference-INTED 2007 (Marzo. 2007). Valencia: IATED. 300.

SANCHO, M.; GARCIA-FAYOS, B.; ARNAL, J.M. (2015).”Tracking of safety learning in two last years of chemical engineering degree”. Gomez-Chova L., Martí-Belenguer D., Candel-Torres, I.(ed.) . En International Technology, Education and Development Conference-INTED 2015 (Marzo. 2015).Madrid: IATED. 4450-4458

SHALLCROSS, D.C. (2013). "Safety shares in the chemical engineering classroom" en Education for Chemical Engineers, 9, e94.

SHALLCROSS, D.C. (2013). "Safety education through case study presentations" en Education for Chemical Engineers, 8, e12.

UNIVERSITAT POLITECNICA DE VALENCIA. (2015). http://competencias.webs.upv.es 